Bundesgesundheitsbl - Gesundheitsforsch Gesundheitsschutz 2007 · 50:1324-1330 DOI 10.1007/s00103-007-0340-5 Online publiziert: 5. Oktober 2007 (c) Springer Medizin Verlag 2007

\section{Die (Krypto-)Pyrrolurie in der Umweltmedizin: eine valide Diagnose?}

\section{Mitteilung der Kommission „Methoden und Qualitätssicherung in der Umweltmedizin“}

\section{Einleitung}

Pyrrol $\left(\mathrm{C}_{4} \mathrm{H}_{5} \mathrm{~N}\right)$ ist ein stickstoffhaltiger Heteroaromat, der 1834 zuerst aus Steinkohlenteer isoliert wurde. Von „Pyrrolen" wurde auch im Zusammenhang mit dem sog. Mauve-Faktor gesprochen: Vor mehr als 40 Jahren hatte Irvine [1] gezeigt, dass bei Patienten mit psychiatrischen Erkrankungen (Depressionen, Schizophrenie, Suizidalität) im Urin Substanzen ausgeschieden werden, die mit dem Ehrlich-Reagens blau-lila reagieren. Die ausgeschiedenen Substanzen wurden als "Mauve-Factor" (mauve = malvenfarbig, hellviolett) bekannt, und aus dessen Anwesenheit wurde eine neue Krankheit, die „Malvaria“ abgeleitet [2]. Die chromatographischen Eigenschaften stimmten mit denen von Kryptopyrrol (2,4-Dimethyl-3Ethylpyrrol) überein [3]. Es wurde angenommen, dass das Pyrrol möglicherweise aus einem gestörten Auf- oder Abbau von Häm stammt und ein Indikator für Substanzen sei, die bei den betroffenen Patienten die psychiatrischen Symptome hervorrufen. Irvine (1978) meinte später, dass es sich bei der Substanz wohl nicht um Kryptopyrrol handelt, sondern um 2Hydroxyhämopyrrolen-5-on (Hämopyrrollaktam). Andere Autoren fanden gar keinen Hinweis auf die Existenz derartiger Pyrrole im Urin $[4,5]$.

Einer der wichtigsten Vertreter der Pyrrol-Hypothese war C.C. Pfeiffer. Er entwarf ein biochemisches Modell, wonach Pyrrol eine Bindung mit Pyrido- xalphosphat (Vitamin B6) eingeht und zusätzlich Zink bindet [6]. Nach dieser Vorstellung zeigt Pyrrol im Urin nicht nur eine Erkrankung des Häm-Stoffwechsels an, sondern ist auch ein Hinweis für einen Zink- und Pyridoxal-Verlust der Betroffenen.

Weder für die chemische Identität dieses Komplexes noch für eine Assoziation mit Erkrankungen wurden seither wissenschaftlich fundierte Belege präsentiert. Die Forschung über die physiologische Herkunft der Pyrrole und mögliche Assoziationen mit Erkrankungen wie Porphyrie und Psychosen hatte ihren Höhepunkt somit vor etwa 25 Jahren und endete damals ohne fassbare Ergebnisse.

Dennoch wird bis heute von einigen Umweltmedizinern im Umkreis von „Klinischen Ökologen“ und von Vertretern der „Orthomolekularen Medizin“ die Diagnose „Pyrrolurie“ gestellt, wenn vermehrt Kryptopyrrol oder verwandte Verbindungen im Urin ausgeschieden werden. Nach diesen Vorstellungen korreliert die vermehrte Ausscheidung mit vielerlei Beschwerden und Krankheiten. Eine Besserung wird durch Gabe von Mikronährstoffen, besonders Vitamin B6 und Zink versprochen. Diagnose- und Therapieangebote im Internet sind jedoch nicht einheitlich.

Beim Online-Lexikon Wikipedia sind die Stichworte „Pyrrolurie“ und Kryptopyrrolurie" gelistet. Im Internet findet man zahlreiche Homepages der Selbsthilfegruppen sowie von Therapeuten und La- boratorien, die ihre Dienste anbieten. Dort werden mühelos pathogenetische Ketten zwischen dem (nicht nachgewiesenen) Mangel an Vitamin B6 und Zink, den sich daraus ergebenden Störungen im Intermediärstoffwechsel einschließlich Redoxsystem und Hypothesen zur Entstehung von Autismus, Alzheimer, AufmerksamkeitsHyperaktivitätssyndrom (ADHS), Allergien, Autoimmunerkrankungen und dem Einfluss von z. B. Schwermetallen aus der Umwelt geknüpft. Diese Ausführungen müssen für Eltern von verhaltensauffälligen Kindern, für chronisch Kranke und für Personen mit Symptomen, für die keine Ursache gefunden werden kann, sehr überzeugend wirken, zumal gleichzeitig Hilfe in Form von „orthomolekularen“ Produkten angeboten wird. Der Hinweis, dass die „Schulmedizin“ hier versagt habe und dass dem Geschehen durch eine einfache Urinuntersuchung jetzt ein Name gegeben werden könne, dürfte für Laien/ Patienten überzeugend wirken.

\section{Die vielen Namen}

Einige Autoren stellen bei der Namensgebung die Rotfärbung des reagenzbehandelten Urins (Malvarie) in den Vordergrund, andere die vermutete chemische Verbindung (Pyrrol, Krypto-, Hämopyrrol, Hämopyrrolactam). Daneben gibt es unterschiedliche Schreibweisen. Aber im Prinzip bezeichnen die in der Tabelle angegebenen 19 Namen alle dasselbe Phänomen (• Übersicht 1). 
Übersicht 1

Verschiedene Namen und Synonyme für Pyrrolurie
- Pyrolurie
- Pyrrolurie
- Pyrrollurie
- Kryptopyrolurie
- Kryptopyrrolurie
- Cryptopyrrolurie
- Hämopyrrolurie
- Hemopyrrolurie
- Hämopyrrolactamurie
- Hämopyrrolaktamurie
- Haemopyrrolactamurie
- Haemopyrrollactamurie
- Haemopyrrollactamie
- Malvarie
- Pyroluria (engl)
- Pyrroluria (engl)
- Malvaria (engl)
- Kryptopyroluria (engl)
Kryptopyrroluria (engl)

\section{Zur Identität der Pyrrole}

Die Untersuchungen zur „Pyrrolurie“ wurden im Wesentlichen von der Arbeitsgruppe um Irvine vorangetrieben. Im Jahre 1961 haben sie einen Stoff im Urin beschrieben, der mit dem Ehrlich-Reagenz (p-Dimethylaminobenzaldehyd) blaulila reagiert und wegen seiner Farbe als „Mauve-Factor" (mauve = hellviolett) bezeichnet wurde [1]. Der Nachweis geschah mit kolorimetrischen und photometrischen Methoden. Ehrlich-Reagenz reagiert mit Aminen, Indolen und Pyrrolen (rot, blau), mit Harnstoff, Xanthuren- und Urocaninsäure (gelb, orange) sowie mit Citrullin (gelb). Eine vorherige Extraktion mit organischen Lösungsmitteln und ein Zusatz von Ascorbinsäure zur Urinprobe sollen die Interferenz mit anderen Substanzen verhindern. Die Autoren fanden, dass der Mauve-Faktor hinsichtlich seines chromatographischen Verhaltens (Papier- und Dünnschichtchromatographie) und seiner Färbeeigenschaften mit der Substanz Kryptopyrrol (2,4-Dimethyl-3-Ethylpyrrol) übereinstimmte [3], und vermuteten, dass sie als Erste Kryptopyrrol (• Abb. 1) im Urin des Menschen nachgewiesen haben. Wenig später wurde die Identifikation des Mauve-Faktors als Kryptopyrrol bestätigt $[7,8]$.

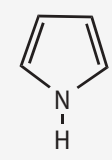

Pyrrol

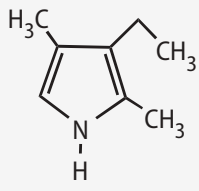

Kryptopyrrol<smiles>CCc1c[nH]c(C)c1C</smiles>

Hemopyrrol

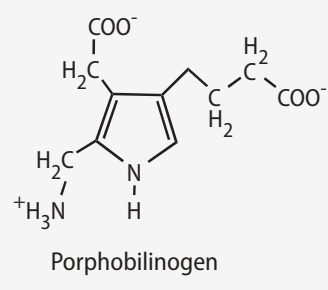

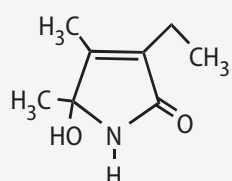

Hydroxyhemopyrrollactam

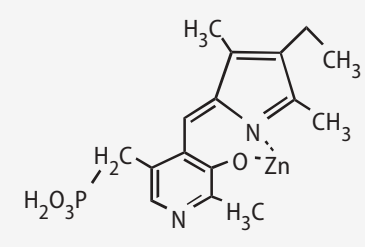

Hypothetischer Komplex nach Pfeiffer et al.,1974

Abb. $1 \Delta$ Pyrrole

Da als Ursache für das Erscheinen von Pyrrolen im Urin eine Störung des Porphyrin-Metabolismus in Erwägung gezogen wurde, wurde der Urin von PorphyriePatienten untersucht. Es wurde ein Pyrrol gefunden, das sich chromatographisch wie Kryptopyrrol verhielt, aber auch mit der strukturverwandten Verbindung Hämopyrrol identisch sein konnte [9]. Der Stand der Kenntnisse bis zum Jahre 1974 und die Diskussion über die Instabilität der Stoffe wurde in einer Übersichtsarbeit von Irvine zusammengefasst [10]. Die Analytik wurde dadurch erschwert, dass die Substanz nicht stabil war [3] und sich spontan in 5 oder mehr Einzelsubtanzen aufteilte [10]. Als deswegen Hämopyrrol und Kryptopyrrol in gezielten In-vitroVersuchsansätzen oxidiert wurden und die Produkte mit den damals verfügbaren Methoden identifiziert wurden, ergab sich, dass es sich bei den Oxidationsprodukten um verschiedene Pyrrol-Lactame handelt, also Pyrrol-Ringe, bei denen am C-Atom neben dem Ringstickstoff ein Sauerstoffatom in Doppelbindung steht $[11,12,13]$. Diese Lactame wurden von einigen Autoren als die eigentlichen Nachweisprodukte im Urin angesehen.

Pfeiffer und Mitarbeiter postulierten, dass es sich bei dem Mauve-Faktor um ein endogenes Pyrrol handelt, das mit Pyridoxalphosphat (Vitamin B6) eine chemische Verbindung eingeht (Schiffsche Base), die mit Zink einen Komplex bildet $(\bullet$ Abb. 1) und im Urin ausgeschieden wird [6]. Dadurch soll das Pyrrol zu einer Verarmung des Körpers an Vitamin B6 und Zink führen [6]. Ein Beleg fehlt.

Mit dieser Hypothese endete die Forschung um die Identität des Mauve-Faktors, obwohl in den 197oer-Jahren die chemische Spurenanalytik einen rasanten Aufschwung erlebte. Eine sichere Bestätigung der chemischen Identität des Pyrrols fehlt bis heute.

\section{Zur möglichen Herkunft von Pyrrolen}

\section{Porphyrinsynthese}

Pyrrole sind chemische Ringverbindungen mit 5 Atomen, davon ein Ring-Stickstoff, und 2 Doppelbindungen. Solche Verbindungen kommen in der menschlichen Biochemie beim Aufbau (Porphobilinogen, - Abb. 1) und Abbau des roten Blutfarbstoffes vor. So wurde vermutet, dass Pyrrole im Urin im Rahmen des biochemischen Syntheseweges bei der Porphyrinsynthese anfallen könnten $[13,14]$.

In den ersten Biosyntheseschritten von Häm entsteht aus Glycin und SuccinylCoA mithilfe des Enzyms 5-Aminolaevulinatsynthase, die Pyridoxylphosphat als Coenzym benötigt, 5-Aminolaevulinat (- Abb. 2, nach [15]). Diese Reaktion ist endproduktkontrolliert, d. h., die Synthese wird eingestellt bei ausreichendem Vorhandensein von Häm. Die Verschmelzung von zwei Molekülen 5-Aminolaevulinsäure zu Porphobilinogen wird durch eine spezifische zinkhaltige Dehydratase 


\section{Empfehlung des Robert Koch-Instituts}

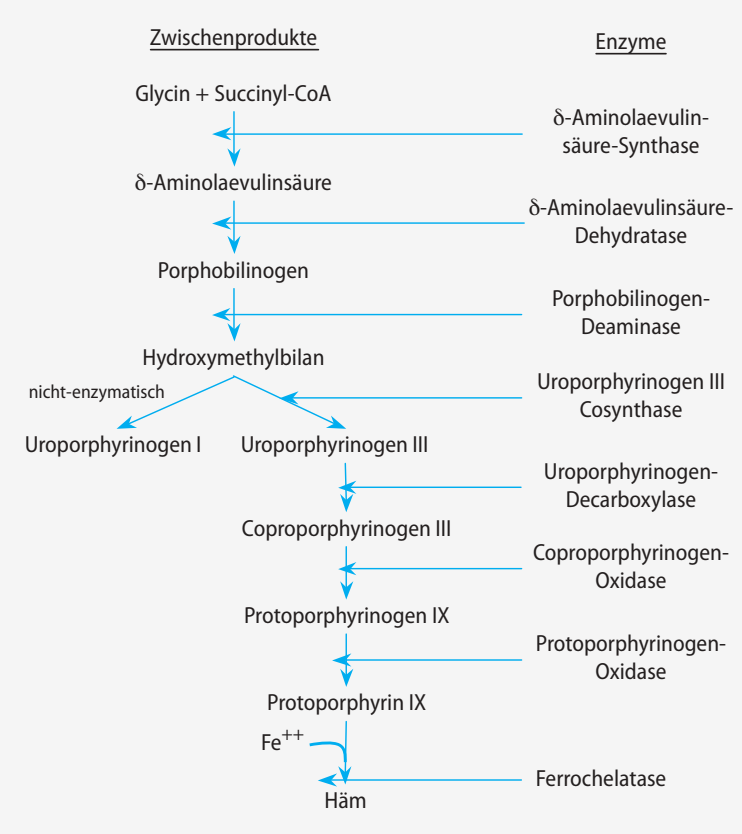

Zwischenprodukte

Glycin + Succinyl-CoA

$<$

phobilinog

xymethylbilan
Krankheiten

Sideroblastische Anämie

(x-chromosomal)

Blei-Intoxikation

ALAD-defiziente Porphyrie (autosomal-rezessiv)

Akute intermittierende Porphyrie (autosomal-dominant)

Kongenitale erythropoetische Porphyrie (autosomal-dominant)

Porphyria cutanea tarda (variabel) Hepatoerythropoetische Porphyrie (autosomal-rezessiv)

Hereditäre Koproporphyrie (autosomal-dominant)

Porphyria variegata (autosomal-dominant)

Erythropoetische Protoporphyrie (autosomal-dominant)

Abb. $2 \Delta$ Porphyrinsynthese, Enzyme, Zwischenprodukte und Krankheiten bei Enzymdefekten (Porphyrien)

katalysiert. Vier Porphobilinogenmoleküle kondensieren unter Abspaltung von Ammoniumionen durch die Porphobilinogendeaminase schrittweise zu einem linearen Tetrapyrrol (Hydroxymethylbilan), das durch Ringbildung mithilfe der Uroporphorinogen-III-Co-Synthase in Uroporphyrinoge III, einer Vorstufe von Häm, umgeformt wird.

Verschiedene Untersuchungen über den möglichen Zusammenhang zwischen Porphyrien und dem Erscheinen von Pyrrolen im Urin wurden durchgeführt: Bei Patienten mit Enzymdefekten der Hämsynthese ist von einigen Autoren die Ausscheidung von (Krypto-),Pyrrolen" beschrieben worden, vor allem in der akuten Erkrankungsphase [4, 9, 13, 16, 17, 18]. Die Gabe von Kryptopyrrol erhöhte die Porphyrin-Ausscheidung im Urin der Ratte [16]. Und die Zugabe von Hämopyrrol und Hämopyrrol-Lactam zu einer Bakterienkultur führte zur vermehrten Bildung von Porphyrin [19]. Neuere Literatur, die den Zusammenhang bestätigen könnte, gibt es nicht.

\section{Häm-Abbau}

Der Abbau von Häm andererseits verläuft über Biliverdin (Enzym ist Hämoxygenase) zu Bilirubin (Biliverdinreduktase). Bilirubin wird in der Leber an Glukuron- säure gekoppelt und als Bilirubindiglukuronid über die Galle ausgeschieden. Im Darm kann Bilirubin durch Reduktion in Mesobilirubin, Urobilinogen und nach Dehydrierung in die Stuhlfarbstoffe Stercobilirubin und Urobilin und andere Abbauprodukte umgewandelt werden. Darunter sind auch Produkte, die aus 2 Pyrrolkernen bestehen [20]. Verantwortlich für diesen Abbau sind im Wesentlichen Darmbakterien. Ein Teil der Gallenfarbstoffe wird im Darm wieder resorbiert und über die Pfortader der Leber zugeführt (enterohepatischer Kreislauf), der größte Teil wird jedoch mit dem Stuhl ausgeschieden [21]. Es wäre theoretisch denkbar, ist aber nicht bekannt, dass Pyrrole wie das „Kryptopyrrol“ im Rahmen des Häm-Abbaus z. B. als Produkt von Darmbakterien entstehen könnten, das dann teilweise resorbiert und über die Niere ausgeschieden werden könnte.

\section{Andere mögliche Quellen}

Es gab auch Hinweise und Vermutungen, dass es sich beim Mauve-Faktor um Stoffe aus der Nahrung handeln könnte [22] oder um Metabolite von Medikamenten, die bei Psychosen verabreicht werden [23]. Aber diese Hinweise wurden nicht konsequent weiter untersucht.

\section{Bezug zu Krankheiten}

\section{Porphyrien}

Defekte an den einzelnen enzymatischen Schritten der Hämsynthese führen zu den verschiedenen Formen der hereditären Porphyrie [24, 25] (• Tabelle 1). Die Störungen der Porphyrinsynthese können oft lange kompensiert werden, und Symptome treten dann erst im Erwachsenenalter oder überhaupt nicht auf. Die häufigste Porphyrieform ist die variabel vererbte Porphyria cutanea tarda, bedingt durch einen Mangel an Uroporphyrinogen-Decarboxylase mit wechselnder Lichtempfindlichkeit der Haut und in einigen Fällen Hepatopathie mit Eisenspeicherung. Die homozygote Form dieses Enzymmangels führt zur hepatoerythropoetischen Porphyrie mit hochgradiger Lichtempfindlichkeit und variablen Leberfunktionsstörungen. Ein Mangel an Porphobilinogendeaminase ist die Ursache der akuten intermittierenden Porphyrie, die autosomal dominant vererbt wird und, ausgelöst durch Hormone, Arzneimittel und Ernährungsfaktoren, neurologische Symptome ohne Lichtempfindlichkeit hervorruft. Das Enzym, das Hydroxymethylbilan umwandelt, fehlt bei der kongenitalen erythropoetischen Porphyrie. Diese Krankheit geht mit der Ausscheidung anderer (symmetrischer) Porphyrine einher, einer Zerstörung von roten Blutzellen, einer Rotfärbung des Urins, einer Fluoreszenz der Zähne im ultravioletten Licht und einer schweren Überempfindlichkeit der Haut für Licht, die zu Narben und Wachstumsstörungen und einer verkürzten Lebenserwartung führt. Die Störung wird autosomal rezessiv vererbt.

Die jeweils charakteristischen und sehr vielfältigen Symptome werden mindestens teilweise durch die toxischen Effekte von pathologisch erhöhten Stoffwechselprodukten aus der Porphyrinsynthese ausgelöst [26]. Expositionen gegenüber üblichen Dosen von Alkohol, Arzneimitteln, Hormonen oder Nahrungsmittelkomponenten können zum akuten Ausbruch einer Porphyrie mit ihren vielfältigen Symptomen führen [27, 28]. Die Symptome können bei Weglassen des auslösenden Faktors reversibel sein. Be- 
Tabelle 1

Obere tolerierbare tägliche Zufuhrmengen (UI) von Vitaminen und Mineralstoffen für Erwachsene [61]

\begin{tabular}{lrlr} 
Nährstoff & \multicolumn{1}{c}{ UI } & Nährstoff & UI \\
Selen $(\mu \mathrm{g})$ & 300 & Vitamin A (mg RE) & 3 \\
\hline Molybdän $(\mu \mathrm{g})$ & 600 & Vitamin D $(\mu \mathrm{g})$ & 50 \\
\hline Magnesium $(\mathrm{mg})$ & 250 & Vitamin E $(\mathrm{mg} \mathrm{TE})$ & 300 \\
\hline Jod $(\mu \mathrm{g})$ & 600 & Nikotinsäure $(\mathrm{mg})$ & 10 \\
\hline Zink $(\mathrm{mg})$ & 25 & Nikotinamid $(\mathrm{mg})$ & 900 \\
\hline Kupfer $(\mathrm{mg})$ & 5 & Folsäure $(\mathrm{mg})$ & 1 \\
\hline Calcium $(\mathrm{mg})$ & 2500 & Vitamin B6 & 25 \\
\hline Fluorid $(\mathrm{mg})$ & 7 & & \\
\hline Bor $(\mathrm{mg})$ & 10 & & \\
\hline RE Retinol-Äquivalent; TE alpha-Tocopherol-Äquivalente & \\
\hline
\end{tabular}

stimmte Schadstoffe (Blei, polybromierte Biphenyle) können nach hoher Exposition auch ohne Vorliegen einer genetischen Disposition die Hämsynthese stören [27, 29, 30].

Weil der Verlauf von Porphyrien durch exogene und wahrscheinlich auch endogene Stoffe beeinflusst wird, erschiene es theoretisch möglich, dass auch Pyrrole hierbei eine Rolle spielen könnten. Aber der früher vermutete Zusammenhang zwischen Pyrrolurie und Porphyrie $[9,16]$ wurde später durch Gorchein infrage gestellt [17] und danach nicht weiterverfolgt. Zwar spielt auch heute noch die Methodik des Nachweises von Porphobilinogen unter Verwendung von Ehrlichs Reagenz eine Rolle bei der Differenzialdiagnose von Porphyrien [31]. Aber die Messung des Mauve-Faktors gehört nicht zum diagnostischen Repertoire.

\section{Chemikalien-Empfindlichkeit}

Weil chemische Stoffe wie Alkohol, Arzneimittel, Hormone, Nahrungsmittel und Schadstoffe einen akuten Ausbruch der Krankheitssymptome einer Porphyrie auslösen können, haben einige Autoren die Vermutung aufgestellt, dass dem Phänomen "multiple chemical sensitivity" (MCS) eine Porphyrie zugrunde liegt, die durch eine Exposition gegenüber Chemikalien ausgelöst wird [32, 33, 34, 35, 36]. Auch wurde eine Assoziation zwischen abnormem Porphyrin-Metabolismus und chronic fatigue syndrom (CFS) vermutet [37].
Etwa zur gleichen Zeit gab es Hinweise, dass Porphyrien viel häufiger seien, als bis dahin angenommen $[35,36,38]$. Weitergehende Analysen zeigten aber, dass die berichteten hohen Porphyrinurieraten artifiziell durch eine zu enge Interpretation von Labordaten bedingt waren [39, 40, 41]. In einer Übersichtsarbeit kamen 13 Experten zu dem Schluss, dass es nicht gerechtfertigt sei, MCS auf eine Störung des Porphyrin-Metabolismus zurückzuführen. Gleichzeitig erinnerten sie daran, dass Kliniker bei porphyrieartigen Symptomen auch an die Diagnose „Porphyrie“ denken sollten $[27]$.

\section{Psychose und Schizophrenie}

In den 1960er-Jahren des letzten Jahrhunderts wurde intensiv nach möglichen biochemischen Markern für psychische Erkrankungen geforscht. 1961 fanden Irvine und Mitarbeiter, dass der MauveFaktor mit Psychosen assoziiert ist [1]. 1963 schlugen Hoffer und Mitarbeiter vor, dass Personen, die den Mauve-Faktor ausscheiden, an einer psychischen Krankheit leiden, die sie als Malvaria bezeichneten [2, 42]. Assoziationen zwischen dem MauveFaktor/Kryptopyrrol und psychiatrischen Erkrankungen, besonders Schizophrenien, wurden von verschiedenen Autoren beschrieben $[18,23,43,44]$. Nach Auffassung von Irvine und Mitarbeiter war der Mauve-Faktor bei 30-60\% von psychiatrischen Patienten nachweisbar [3]. Die Arbeitsgruppe von Pfeiffer postulierte eine chemische Verwandtschaft zwischen dem Pyrrol und einem Geruchsstoff, der bei schizophrenen Patienten vermehrt ausgeschieden wird [45]. Allerdings wurden mit einer damals modernen gaschromatischen Methode keine erhöhten Urinwerte an Hämopyrrol oder Kryptopyrrol bei schizophrenen Patienten gefunden [4, 5]. Auch andere Autoren fanden, dass die Pyrrolurie ein schlechter Marker für Schizophrenie sei [17, 22]. Es gab auch Hinweise, dass es sich bei den farbgebenden Stoffen des Mauve-Faktors im Urin der Schizophreniepatienten um Metabolite von Psychopharmaka handelt [23].

Die Frage, ob die Pyrrole eine Intoxikationspsychose auslösen können, wurde in Tierversuchen untersucht. Die Verabreichung hoher Dosen von Kryptopyrrol an Ratten führte zu Ataxie, Hyperventilation, Bewegungsarmut und Katalepsie sowie EEG-Veränderungen, die Ähnlichkeiten mit den hypersynchronen EEG-Mustern nach Einnahme halluzinatorischer Stoffe aufwiesen [46]. Pharmakologische Untersuchungen $\mathrm{zu}$ neurologischen Wirkungen von Kyptopyrrol und seiner Oxidationsprodukte (Lactame) wurden in Nagetieren durchgeführt. Die Befunde sprachen dafür, dass die Substanzen zentral depressiv wirken [47], was sich nicht mit der Hypothese deckte, dass das Pyrrol möglicherweise der auslösende Faktor für Schizophrenie wäre.

Dessen ungeachtet, wurde das psychiatrisch-neurologische Symptomenspektrum von Verfechtern der PyrrolurieHypothese erweitert auf Störungen des Kurzzeitgedächtnisses, des Traumerinnerungsvermögens und der zwischenmenschlichen Kommunikation, auf das Aufmerksamkeitsdefizit-Hyperaktivitätssyndrom (ADHS), auf zerebrale Abbauprozesse mit neurologischen Funktionsstörungen, wie z. B. Verschlechterung der Handschrift, Schlafstörungen, Wahrnehmungsstörungen, rheumatologische Beschwerden und „Autoimmunphänomene". Dies wird durch keine wissenschaftliche Literatur gestützt. Die Anhänger der Kryptopyrrolurie-Hypothese betonen, dass ein kausaler Zusammenhang bisher nicht bewiesen wurde, behaupten aber gleichzeitig, dass $10 \%$ der Bevölkerung eine "Störung der Hämsynthese“ aufweisen, und dass der Nachweis einer abnorm gesteigerten Pyrrolausscheidung 
im Urin $(>15 \mu \mathrm{g} / \mathrm{dl}$ oder $1 \mu \mathrm{mol} / \mathrm{l}) \mathrm{die}$ Diagnose Kryptopyrrolurie zuverlässig erlaube (Internetrecherche).

\section{Nährstoffmangel}

Nach der Hypothese von Pfeiffer werden „überschüssige“ Pyrrole komplexiert an Pyridoxalphosphat und chelatiert mit Zink (Hämopyrrollactam) vermehrt im Urin ausgeschieden, was zu Vitamin-B6und Zinkmangel führe [6, 48]. Durch die behauptete vermehrte Ausscheidung von Zink und Vitamin B6 komme es zu Mangelsymptomen, wodurch sich das Symptomenspektrum der Kryptopyrrolurie noch erweitere: Infektionsneigung, Wundheilungsstörungen, Haarausfall, brüchige Nägel, rissige Haut, Hautstriae, Acne vulgaris, Weißflecken der Nägel, Kopfschmerzen, Angstzustände, Panikattacken, morgendliches Erbrechen, Schwangerschaftserbrechen, Karpaltunnelsyndrom, Zahnwachstumsstörungen, prämenstruelles Syndrom, Infertilität und Knochen-, Gelenk- und Muskelbeschwerden.

Der behauptete Vitamin-B6- und Zinkmangel wurde nie durch Laboruntersuchungen belegt. Cruz fand bei einigen Patienten keinen Hinweis auf einen Mangel [22]. Selbst wenn ein Pyrrol-VitaminB6-Zink-Komplex ausgeschieden würde, zeigen Berechnungen, dass dies keinen Mangel mit sich bringen sollte. Denn die Ausscheidung von $1 \mu \mathrm{mol}$ Hämopyrrol (entspricht 123 Mikrogramm; Molekulargewicht 123) würde nur den Verlust von $1 \mu \mathrm{mol}$ Vitamin B6 und $1 \mu \mathrm{mol}$ Zink mit sich bringen, das heißt ein Sechstel der empfohlenen täglichen Zufuhr von Vitamin B6 (Molekulargewicht 170) bzw. ein 153stel der empfohlenen täglichen Zufuhr von Zink (Atomgewicht 65). Außerdem wären bei einem Mangel an Zink unspezifische Symptome zu erwarten wie Haarausfall, Durchfall, Appetitlosigkeit, Haut- und Schleimhautläsionen und bei Kindern Wachstumsrückstand sowie als mehr spezifisches Zeichen eine Erniedrigung der Aktivität der alkalischen Phosphatase im Serum. Bei einem Mangel an Vitamin B6 dagegen wäre mit wiederum unspezifischen klinischen Symptomen wie seborrhoischer Dermatitis, mikrozytärer Anämie, Depressionen und in schweren
Fällen Krämpfen zu rechnen und im Laboratorium sollten niedrige Vitaminspiegel und die vermehrte Ausscheidung von Tryptophanmetaboliten (Xanthurensäure) und Methioninmetaboliten (Homocystin, Cystathionin) nachzuweisen sein.

\section{Heutige Darstellung der Pyrrolurie durch Befürworter}

Eine aktuelle wissenschaftliche Literatur zu Pyrrolurie gibt es nicht, lediglich einige kürzere Darstellungen von Vertretern der Pyrroluriethese in diversen Zeitschriften, die z. T. umweltmedizinischen Bezug haben $[49,50,51]$, sowie in jüngster Zeit zwei kritische Abhandlungen in niederländischer Sprache $[52,53]$. Aber im Internet findet man zahlreiche Angebote zur Messung von Pyrrolurie und ggf. zur Behandlung der Pyrrolurie, vor allem mit Methoden der orthomolekularen Therapie.

Viele der dort konstruierten Zusammenhänge sind in ähnlicher Weise schon vor 30 Jahren von Pfeiffer formuliert worden $[6,48]$. Um dies zu demonstrieren, wird aus Pfeiffers Buch „Nutrition and Mental Illness; An Orthomolecular Approach to Balancing Body Chemistry“ [54] ein Auszug aus dem Kapitel „B6 und Zink - der fehlende Zusammenhang" in deutscher Übersetzung gegeben:

„Vielleicht die wichtigste Entdeckung für die Ernährungsbehandlung von psychisch kranken Menschen besteht darin, dass viele Depressive und seelisch kranke Menschen einen Mangel an Vitamin B6 und Zink aufweisen. Aber dies ist kein gewöhnlicher Mangel, den man einfach durch vermehrten Verzehr von VitaminB6- und zinkreichen Nahrungsmitteln beheben könnte. Er steht in Beziehung zur abnormen Bildung einer Gruppe von Chemikalien, die als ,Pyrrole bezeichnet werden. Ein Patient mit hohem Pyrrolspiegel im Urin benötigt mehr Vitamin B6 und Zink, da die Pyrrole dem Körper diese essenziellen Nährstoffe entziehen. Etwa $30 \%$ der Schizophrenen und $11 \%$ der normalen Bevölkerung haben ,Pyrrolurie،“”

\section{Selbstdiagnose der Pyrrolurie}

Die folgenden Hinweise von Pfeiffer [54] sind im Internet verbreitet und ermögli- chen es Patienten oder deren Familienangehörigen, allein durch Beantworten einer Frageliste selbst die Verdachtsdiagnose „Pyrrolurie“ zu stellen. Die Symptome sind so allgemein, dass sie für fast jeden Menschen, dem es nicht gut geht, zutreffen:

- Intoleranz gegenüber bestimmten proteinreichen Nahrungsmitteln, Alkohol oder Arzneimittel,

- eindeutiger Mundgeruch und Körpergeruch,

- morgendliche Übelkeit und Verstopfung,

- schlechte Traumerinnerung,

- eng sitzende obere Schneidezähne,

- weiße Flecken auf den Fingernägeln,

- bleiche, sonnenlichtempfindiche Haut,

- häufige Schmerzen im Oberbauch,

- häufige obere Atemwegsinfekte,

- Dehnungsstreifen der Haut (Stretch marks),

— unregelmäßige Menstruation oder Impotenz,

- einer der genannten Befunde unter Stress,

- sie gehören zu einer Familie mit nur Mädchen, die einander ähnlich sehen.

Auf Internetseiten für Patienten werden zusätzliche Zusammenhänge angegeben. So findet man die Aussage, die Pyrrolurie werde bei $70 \%$ aller Patienten mit Trisomie 21, bei $50 \%$ aller Autismuspatienten und $30 \%$ aller Patienten mit ADHS nachgewiesen. Alle Pyrroluriesymptome würden durch Stress verstärkt. Auch werden die Häufigkeiten der Symptome angegeben von Patienten die mehr als $1 \mu \mathrm{mol}$ Pyrrol/1 im Urin ausschieden: Blutarmut $60 \%$, Blässe $68 \%$, Barbituratunverträglichkeit $8 \%$, aufgedreht als Kind 34\%, Durchfall $54 \%$, Verstopfung $42 \%$, helle Stuhlfarbe $46 \%$, Bauchschmerzen $52 \%$, Weißfleckennägel $22 \%$, Antibiotikaeinnahme $44 \%$, Schlafstörungen $60 \%$, trockene Haut $60 \%$, Muskelzittern $60 \%$, schlechter Atem $34 \%$, dominante Mutter $28 \%$ und viele andere. In diesen Aufzählungen findet sich also für fast jeden ein Anlass, eine Urinuntersuchung vornehmen zu lassen. Belege für diese Behauptungen lassen sich nicht finden. 
Heutige Behandlungsangebote bei Pyrrolurie

In den 1970er-Jahren begann die Ära der Megavitamin-Dosierung zur Vorbeugung und Behandlung verschiedener Krankheiten, wobei der Nobelpreisträger Linus Pauling in führenden Medizinzeitschriften als Befürworter auftrat $[55,56]$. Der Begriff „orthomolekulare Medizin“ wurde eingeführt, ebenso das neue Arbeitsfeld „orthomolekulare Psychiatrie“ $[6,57,58,59]$, das als Außenseitermethode angesehen werden kann. Dieser Zweig der biologischen Psychiatrie sah die Ursachen aller Psychosen in einem biochemischen Ungleichgewicht im Gehirn, welches durch Nahrungsmittel, vor allem durch hoch dosierte Vitamine und Spurenelemente behandelbar sei.

Auch die Pyrrolurie sei durch Vitamingaben behandelbar, zumal sie infolge der Ausscheidung eines Pyrrol-Vitamin-B6Zink-Komplexes zur Verarmung des Körpers an Vitamin B6 und Zink führe.

Dementsprechend empfiehlt Pfeiffer [54] für Personen, welche die weiter oben zitierten Pyrroluriesymptome aufweisen, folgende Behandlung, die in den meisten Fällen schon bald zum Verschwinden der Symptome führe: 1. morgendlich Vitamin B6 in einer Dosis, unter der Nachtträume wieder erinnert werden (nicht über $2000 \mathrm{mg}$ ); 2. Zink (als Glukonat), $30 \mathrm{mg}$ morgens und abends; 3. Mangan (als Glukonat) $10 \mathrm{mg}$, morgens und abends.

In der Regel enthalten die mit der Indikation „Pyrrolurie“ im Internet angebotenen Produkte hohe Dosen an Vitamin B6 und Zink, ggf. auch andere essenzielle und nicht-essenzielle Nährstoffe. Die Dosen liegen $z$. T. weit über den oberen tolerierbaren Dosen (upper limits) (• Tabelle 1).

\section{Nachweismethode}

Für den Nachweis des Mauve-Faktors und der verschiedenen Pyrrole wurden vor 30 Jahren papierchromatographische Methoden, dünnschichtchromatographische Methoden und später GC-MS-Methoden beschrieben. Damals erwies sich der Mauve-Faktor als instabil. Über die Identität gab es unterschiedliche Angaben. Eine zweifelsfreie Identifizierung fand nicht statt. Es gibt keine Standardmethode. Einige Labors geben an, Kryptopyrrol zu messen, andere Hämopyrrol, andere Kryptopyrrollactam oder den PyridoxalZink-Komplex dieser Verbindung. Damit entfallen wichtige Möglichkeiten der internen und externen analytischen Qualitätskontrolle. $\mathrm{Zu}$ diesen würden $\mathrm{z}$. B. Angaben über die Einheitlichkeit des Analyten, eingesetzte Standards, Reproduzierbarkeit und Ergebnisse aus Ringversuchen gehören. Verlässliche Informationen über die intraindividuellen und interindividuellen Schwankungen der Pyrrolausscheidung stehen nicht zur Verfügung. Aber selbst wenn der Mauve-Faktor richtig gemessen werden könnte, so fehlt doch, wie oben beschrieben, der Bezug zu einer Krankheit.

\section{Zusammenfassung und Bewertung}

Die in der früheren Literatur geäußerten Hypothesen über einen Zusammenhang zwischen Pyrrolen im Urin und verschiedenen Erkrankungen wurden nicht bestätigt. Außerdem sind Herkunft und chemische Identität der in Rede stehenden Stoffe nicht hinreichend geklärt. Neuere wissenschaftliche Literatur fehlt. Somit kann bei einer eventuell erhöhten Ausscheidung des Mauve-Faktors oder von Pyrrolen derzeit nicht auf eine Stoffwechselstörung oder eine Erkrankung zurückgeschlossen werden. Eine darauf aufbauende Diagnose oder gar Therapieform entbehrt beim heutigen Kenntnisstand der wissenschaftlichen Grundlage. Die Pyrrolurie ist ein Befund, der nach wissenschaftlichen Kriterien keiner Krankheit zugeordnet werden kann.

Die Bestimmung von Pyrrolen fällt damit unter Kategorie IV der RKI-Kommission „Methoden und Qualifikationssicherung in der Umweltmedizin“ („Eine Maßnahme kann nicht empfohlen werden, weil keine ausreichenden, dies begründenden Untersuchungsergebnisse vorliegen [keine eindeutige Identität der Substanz und kein Bezug zu Erkrankungen] und theoretische Überlegungen [sehr breites Symptomenspektrum] gegen eine Anwendung sprechen" [6o]). Untersuchungen zur Diagnose der Pyrrolurie können demnach beim gegenwärtigen Stand der Wissenschaft nicht empfohlen werden.

\section{Federführung}

Prof. Dr. H. Przyrembel, externe Sachverständige (Bundesinstitut für Risikobewertung, BfR), Prof. Dr. M. Schwenk (Tübingen)

\section{Kommissionsmitglieder}

Dr. A. Beyer (Umweltmed. Ambulanz Berlin-Steglitz/Zehlendorf), Prof. Dr. W. Dott (Universitätsklinikum Aachen, Institut für Hygiene und Umweltmedizin), Prof. Dr. H. Drexler (Friedrich-Alexander-Universität Erlangen-Nürnberg, Institut für Arbeits-, Sozial- und Umweltmedizin), Prof. Dr. H. Dunkelberg (Universität Göttingen, Abt. Allg. Hygiene u. Umweltmedizin), Prof. Dr. Th. Eikmann (Universität Gießen, Institut f. Hygiene u. Umweltmedizin), Dr. B. Heinzow (Landesamt für Gesundheit und Arbeitssicherheit Schleswig-Holstein, Dezernat Umweltbezogener Gesundheitsschutz), Prof. Dr. C. Hornberg (Universität Bielefeld, Fakultät für Gesundheitswissenschaften), Prof. Dr. Dr. A.D. Kappos (Frankfurt/Main), Prof. Dr. K.E. von Mühlendahl (Kinderhospital Osnabrück, Gemeinnützige Kinderumwelt $\mathrm{GmbH}$ ), Prof. Dr. D. Nowak (LMU München, Klinikum Innenstadt, Institut u. Poliklinik für Arbeits- und Umweltmedizin), PD Dr. F.-A. Pitten (Institut für Krankenhaushygiene und Infektionskontrolle, Gießen), Dr. W. Stück (Ökologischer Ärztebund/ ISDE, Koblenz), Prof. Dr. M. Schwenk (Tübingen), Dr. R. Suchenwirth (Niedersächsisches Landesgesundheitsamt, Abt. Umweltmedizin/Epidemiologie, Hannover), Prof. Dr. M. Wilhelm (Universität Bochum, Hygiene, Sozial- und Umweltmedizin).

\section{Ständige Gäste}

Dr. N. Englert (Umweltbundesamt, Berlin), Dr. A. Hahn (Bundesinstitut für Risikobewertung, Berlin), Dr. U. Winkler (Bundesministerium für Gesundheit, Referat 332).

\section{Geschäftsstelle im RKI:}

Dr. D. Eis, Dr. U. Wolf. 


\section{Literatur}

1. Irvine DG (1961) Apparently non-indolic Ehrlichpositive substances related to mental illnesses. J Neuropsychiatr 2:292-305

2. Hoffer A, Osmond H (1963) Malvaria: a new psychiatric disease. Acta Psychiatr Scand 39:335-366

3. Irvine DG, Bayne W, Miyashita H, et al. (1969) Identification of kryptopyrrole in human urine and its relation to psychosis. Nature 224(5221):811-883

4. Gendler PL, Duhan HA, Rapoport H (1978) Hemopyrrole and kryptopyrrole are absent from the urine of schizophrenics and normal persons. Clin Chem 24(2):230-233

5. Jacobson SJ, Rapoport H, Ellman GL (1975) The nonoccurrence of hemo- and kryptopyrrole in urine of schizophrenics. Biol Psychiatry 10(1): 91-93

6. Pfeiffer CC, Sohler A, Jennery C, et al. (1974) Treatment of pyroluric schizophrenica with large doses of pyridoxine and a dietary supplement of zinc. J Orthomol Psychiatr 3:292-300

7. Irvine DG, Bayne W, Majer JR (1970) Autotransfer chromatography combined with mass spectroscopy, for the characterization of pyrroles and indoles. J Chromatogr 48(2):334-342

8. Sohler A, Beck R, Noval JJ (1970) Mauve factor re-identified as 2,4-dimethyl-3-ethylpyrrole and its sedative effect on the CNS. Nature 228(5278):1318-1320

9. Irvine DG, Wetterberg L (1972) Kryptopyrrole-like substance in acute intermittent porphyria. Lancet 2(7788):1201

10. Irvine DG (1974) Kryptopyrrole and other monopyrroles in molecular neurobiology. Int Rev Neurobiol 16:145-182

11. Lightner DA, Crandall DC (1973) The dye sensitized photooxygenation of kryptopyrrole. Experientia 29(3):262-264

12. Irvine DG (1976) Autotransfer chromatography in the characterization of pyrroles. Chemistry of multiple-spot phenomena. J Chromatogr 123(1): 69-78

13. Moore MR, Graham DJ (1980) Monopyrroles in porphyria, psychosis and lead exposure. Int J Biochem 12(5-6):827-832

14. Russell CS (1972) Biosynthesis of porphyrins and the origin of the "mauve factor". J Theor Biol 35(2):277-283

15. Thomas L (2005) Porphyrien. In: Thomas L (Hrsg) Labor und Diagnose. TH-Books, Frankfurt: S 646-659

16. Brodie MJ, Graham DJ, Thompson GG, et al. (1976) The porphyrinogenic effects of kryptopyrrole in the rat and the occurrence of urinary kryptopyrrole in human hereditary hepatic porphyria. Clin Sci Mol Med 50(5):431-434

17. Gorchein A (1980) Urine concentration of 3-ethyl5-hydroxy-4,5-dimethyl-delta 3-pyrrolin-2-one (,mauve factor") is not causally related to schizophrenia or to acute intermittent porphyria. Clin Sci (Lond) 58(6):469-476

18. Huszak I, Durko I, Karsai K (1972) Experimental data to the pathogenesis of cryptopyrrole excretion in schizophrenia I. Acta Physiol Acad Sci Hung 42(1):79-86

19. Durko I, Berek I, Huszak I (1975) Effects of kryptopyrrole on porphyrin synthesis in Bacillus subtilis 168. Hoppe Seylers Z Physiol Chem 356(11): 1679-1684

20. Seyfried H, Klicpera M, Leithner C, et al. (1976) Bilirubin metabolism (author's transl). Wien Klin Wochenschr 88(15):477-482
21. Oates PS, West AR (2006) Heme in intestinal epithelial cell turnover, differentiation, detoxification, inflammation, carcinogenesis, absorption and motility. World J Gastroenterol 12(27):4281-4295

22. Cruz R, Vogel WH (1978) Pyroluria: a poor marker in chronic schizophrenia. Am J Psychiatry 135(10): 1239-1240

23. Ellman GL, Jones RT, Rychert RC (1968) Mauve spot and schizophrenia. Am J Psychiatry 125(6): 849-851

24. Brodie MJ, Moore MR, Goldberg A (1977) Enzyme abnormalities in the porphyrias. Lancet 2(8040): 699-701

25. Dombeck TA, Satonik RC (2005) The porphyrias Emerg Med Clin North Am 23(3):885-899

26. Ellefson RD, Ford RE (1996) The porphyrias [corrected]: characteristics and laboratory tests. Regul Toxicol Pharmacol 24(1 Pt 2):119-125

27. Daniell WE, Stockbridge HL, Labbe RF, et al. (1997) Environmental chemical exposures and disturbances of heme synthesis. Environ Health Perspect 105(Suppl 1):37-53

28. Doss MO, Kuhnel A, Gross U (2000) Alcohol and porphyrin metabolism. Alcohol Alcohol 35(2): 109-125

29. Aust SD, Millis CD, Holcomb L (1987) Relationship of basic research in toxicology to environmental standard setting: the case of polybrominated biphenyls in Michigan. Arch Toxicol 60(1-3): 29-237

30. Campbell BC, Brodie MJ, Thompson GG, et al (1977) Alterations in the activity of enzymes of haem biosynthesis in lead poisoning and acute hepatic prophyria. Clin Sci Mol Med 53(4):335-340

31. Gorchein A (2002) Testing for porphobilinogen in urine. Clin Chem 48(3):564-566

32. Maschewsky W (1996) MCS und Porphyrinopathien. Ztg Umweltmed 3:102-106

33. Silbergeld EK (1987) Porphyrins as indicators of chemical injury and exposure. Ann NY Acad Sci 514:170-171

34. Silbergeld EK (1987) Role of altered heme synthesis in chemical injury to the nervous system. Ann N Y Acad Sci 514:297-308

35. Ziem G, McTamney J (1997) Profile of patients with chemical injury and sensitivity. Environ Health Perspect 105(Suppl 2):417-436

36. Baker GP (1995) Porphyria and MCS symptoms overlap - another chemical connection. Townsend Letter Doctors 144:72-73

37. Downey D (1996) Porphyria: a new perspective. Med Hypotheses 46(4):378-382

38. Morton WE (1995) Redefinition of abnormal susceptibility to environmental chemicals. Paper, given at the Second International Congress on Hazardous Waste. Atlanta

39. Hahn M, Bonkovsky HL (1997) Multiple chemica sensitivity syndrome and porphyria. A note of caution and concern. Arch Intern Med 157(3):281-285

40. Mattern SE, Tefferi A (1999) Acute porphyria: the cost of suspicion. Am J Med 107(6):621-623

41. McDonagh AF, Bissell DM (1998) Porphyria and porphyrinology - the past fifteen years. Semin Liver Dis 18(1):3-15

42. Hoffer A (1963) The presence of malvaria in some mentally retarded children. Am J Ment Defic 67:730-732

43. Sohler A, Renz RH, Smith S, et al. (1967) Significance of hydroxyskatole and mauve factor excretion in schizophrenia. Int J Neuropsychiatry 3(4):327-331

44. O'Reilly PO, Ernest M, Hughes G (1965) The incidence of malvaria. Br J Psychiatry 111:741-744
45. Krischer K, Pfeiffer CC (1973) Biochemical relationship between kryptopyrrole (mauve factor and trans-3-methyl-2-hexenoic acid (schizophrenia odor). Res Commun Chem Pathol Pharmacol 5(1):9-15

46. Walker JL (1975) Neurological and behavioral toxicity of kryptopyrrole in the rat. Pharmacol Biochem Behav 3(2):243-250

47. Wetterberg L, Formgren B (1976) Pharmacolog ical and biochemical properties of kryptopyrrole and its oxidation products possibly related to acute intermittent porphyria. Ann Clin Res 8(Suppl 17):162-167

48. Pfeiffer CC (1975) Mental and elemental nutrients. A physican's guide to nutrition and health care. Keats Publishing, New Canaan, Connecticut

49. Prochazka E (2001) Pyrrolurie - das missing link? Umwelt Med Ges 14(4):284-285

50. Kamsteeg J (2002) HPU - eine angeborene Porphyrinopathie. Ztg Umweltmed 10(3):134-135

51. Kuklinski B (2004) Kryptopyrrolurie, nitrosativer Stress und Mitochondropathie. Nährstoff-News Nährstoff-Akademie Salzburg 3:5-8

52. Hessels J, van As S (2006) HPU: Hoe porfyrineonderzoek uit de hand liep. Een wetenschappelijke benadering. Ned Tijdschr Klin Chem Labgeneesk 31:58-64

53. van der Meer JW, van de Kerkhof R, The GK, et al. (2003) Hemopyrrollactamuria (HPU); from spots to pseudo-disease. Ned Tijdschr Geneeskd 147(36):1720-1721

54. Pfeiffer CC (1987) Nutrition and mental illness. An orthomolecular approach to balancing body chemistry. Healing Arts Press, Rochester, Vermont

55. Pauling L (1977) Vitamin homeostasis in the brain and megavitamin therapy. N Engl J Med 297(14):790-791

56. Pauling L (1975) Letter: Megavitamin therapy. Jama 234(2):149

57. Pauling L (1968) Orthomolecular psychiatry. Varying the concentrations of substances normally present in the human body may control menta disease. Science 160(825):265-271

58. Hoffer A (1972) Orthomolecular treatment for schizophrenia. I. J Pract Nurs 22(8):16-19

59. Pratt WA (1975) The orthomolecular approach to schizophrenia. J Am Osteopath Assoc 75(2): 199-205

60. RKI (2001) Mitteilung der Kommission „Methoden und Qualitätssicherung in der Umweltmedizin" am Robert Koch-Institut (RKI). Grundsätze der Bewertung von umweltmedizinischen Methoden. Bundesgesundheitsbl Gesundheitsforsch Gesund heitsschutz 44:519-522

61. EFSA - European Food Safety Authority (2006) Tolerable upper intake levels for vitamins and minerals. EFSA - European Food Safety Authority Scientific Committee on Food; Scientific Panel on Dietetic Products, Nutrition and Allergies. http:// www.efsa.eu.int 